\title{
Aqueye and IqueYe, Very-High-Time-Resolution Photon-Counting Photometers
}

\author{
Cesare Barbieri ${ }^{1}$, Giampiero Naletto ${ }^{1}$, Luca Zampieri ${ }^{2}$, \\ Enrico Verroi ${ }^{1}$, Serena Gradari ${ }^{1}$, Susan Collins ${ }^{3}$ and Andy Shearer ${ }^{3}$ \\ ${ }^{1}$ University of Padova, Italy email: cesare.barbieri@unipd.it \\ ${ }^{2}$ INAF Astronomical Observatory of Padova, Italy, \\ ${ }^{3}$ NUI Galway, Ireland
}

\begin{abstract}
We describe very high-time-resolution photometers capable of tagging the arrival time of each photon with a resolution and accuracy of few hundred picoseconds, for hours of continuous acquisition, and with a dynamic range of more than 6 orders of magnitude. The final goal is the conceptual definition of a "quantum" photometer for the E-ELT, capable of detecting and measuring second-order correlation effects in photon streams from celestial sources. Two prototype units have been built and operated, one for the Asiago 1.8-m telescope (AquEYE) and one for the 3.5-m NTT (IquEYE).Here we will present results obtained by IquEYE on the Crab Nebula pulsar in simultaneous radio observations with Jodrell Bank in December 2009.
\end{abstract}

Keywords. instrumentation: photometers pulsars: individual (Crab)

\section{Introduction}

In 2005 September we completed a study (QuantEYE, the ESO Quantum Eye; Dravins et al. 2005) whose main goal was to demonstrate the possibility of reaching picosecond time resolution (the Heisenberg limit) and sustaining a count rate as high as $1 \mathrm{GHz}$ (needed to bring quantum optics concepts into the astronomical domain), with two main scientific aims in mind:

- to measure the entropy of the light beam through the statistics of photon arrival time, and

- to demonstrate the feasibility of astronomical photon correlation spectroscopy and of a modern version of the Hanbury Brown Twiss Intensity Interferometry (HBTII).

Although the project was conceived for the 100-m Overwhelmingly Large (OWL) telescope, the results reached in that study will be of interest for the ESO E-ELT and other 30-m telescopes too.

\section{Detector}

The most critical point, and the driver for the design of QuantEYE and subsequent prototypes, was the selection of very fast, efficient and accurate photon counting detectors. In order to proceed, we choose Single Photon Avalanche Diodes (SPADs) from the Italian company MPD. These detectors are operated in Geiger, continuous mode, have a 35 ps time resolution through a NIM connector, and sustain count rates as high as $15 \mathrm{MHz}$. Their main drawbacks are the small sensitive area (50-100 $\mu$ m diameter), the lack of a CCD-like array, a $>77$ ns dead time and a 1.5\% afterpulsing probability. 

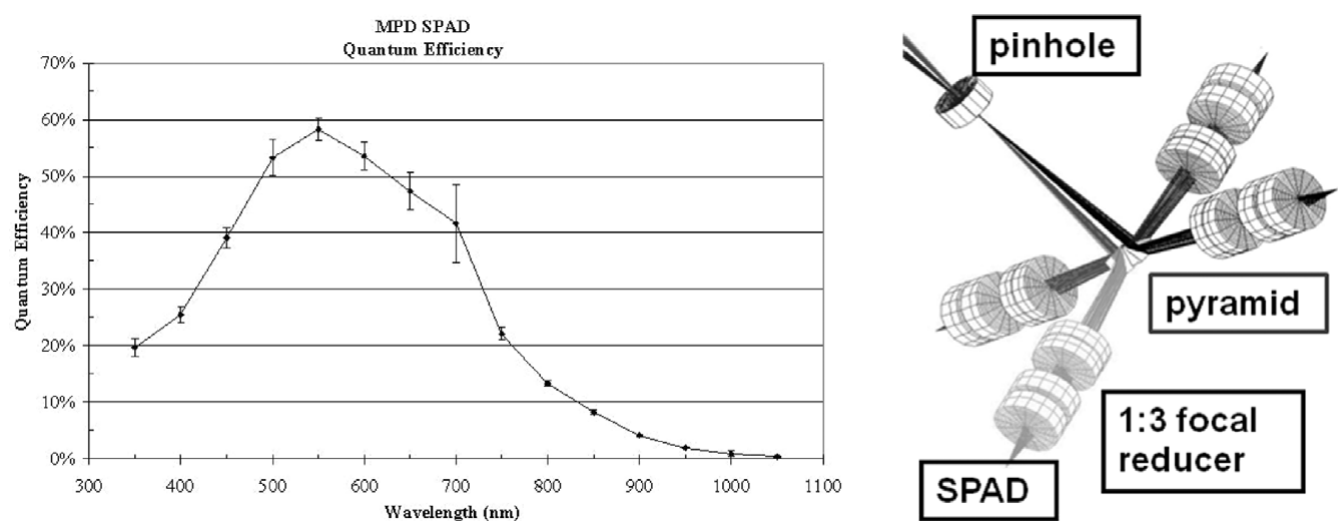

Figure 1. Left: the QE of the MPD SPAD peaks at 55\% around 550nm. Right: The optomechanical concept of AquEYE. The pyramid splits the light beam into four arms, each feeding a SPAD via a 1:3 focal reducer, inside which filters and polarisers can be inserted.

\section{AquEYE}

AquEYE, the Asiago Quantum Eye, was designed as the final stage of AFOSC, the imaging spectrophotometer of the Asiago-Cima Ekar (Italy) 182-cm telescope. AFOSC plays the role of a 1:3 focal reducer. The light beam from AFOSC is divided into four parts by means of a pyramidal mirror. Each beam is then focussed on its own SPAD by another 1:3 focal reducer made by a pair of doublets. This configuration provides a possibility for simultaneous multicolour photometry, and allows the cross-correlation of the 4 sub-apertures, in a conceptual proof of HBTII capabilities.

The pulses from each SPAD are fed into a Time-To-Digital Converter board (TDC), together with the time stamps from a Time and Frequency Unit composed of a GPS receiver and a rubidium clock. The time ticks are treated as light pulses, so the delays inside the hardware and software channels are therefore immaterial. The UTC arrival time of each photon is stored separately for each channel, guaranteeing data integrity for subsequent scientific investigations, where one can either sum up the 4 channels or treat them separately, with arbitrary time binning in units of the basic time unit of the system, namely 24 picoseconds.

\section{IquEYE}

Following the success of AquEYE, IquEYE (Naletto et al. 2009) was built for the ESO 3.5-m NTT at La Silla (Chile). The same basic solutions of 4-way pupil splitting and hardware/software configuration were maintained. The main improvement was a new production batch of MPD SPADs with $100 \mu \mathrm{m}$ diameter, lower dark counts and better engineering. Other improvements were implemented in order to be able to control the instrument remotely from the new LaSilla Control Room.

\section{Crab Observations}

The Crab pulsar was observed with IquEYE in 2009 January and again in 2009 December. The quality of the data is demonstrated in Fig. 2, which shows a short set of individual pulses, with data binned at 0.001 second intervals.

The optical data were folded using the JB radio ephemeris fitted over the observing dates. A specially fitted ephemeris was required, owing to the rapidly varying dispersion 


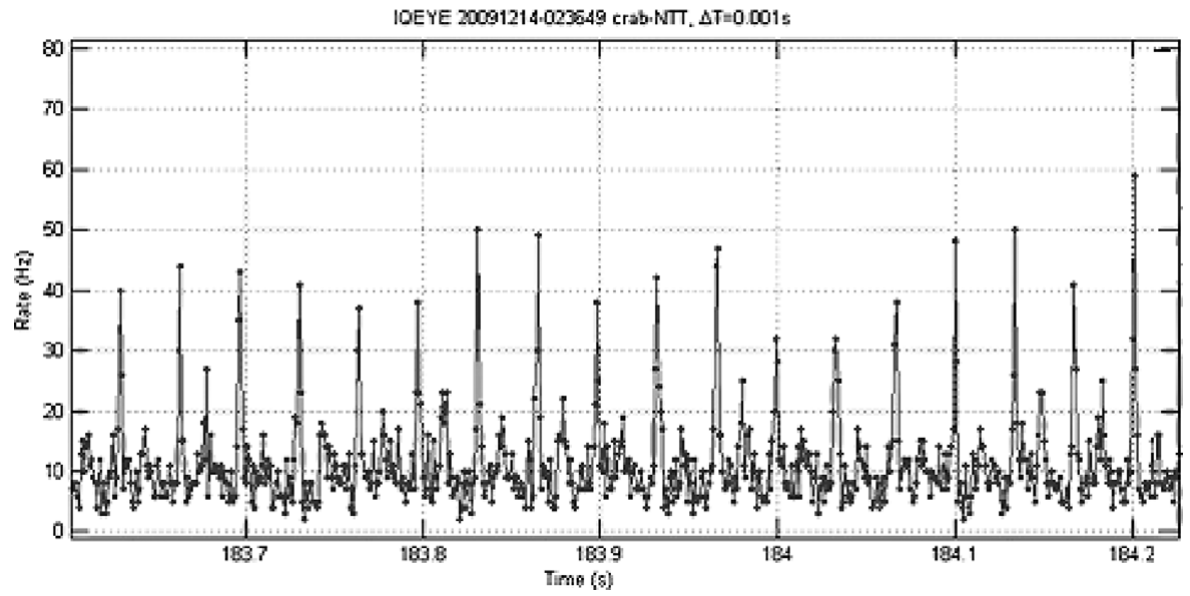

Figure 2. Crab Pulsar Observations: A short section of individual primary and secondary peaks, binned at 0.001 second intervals.

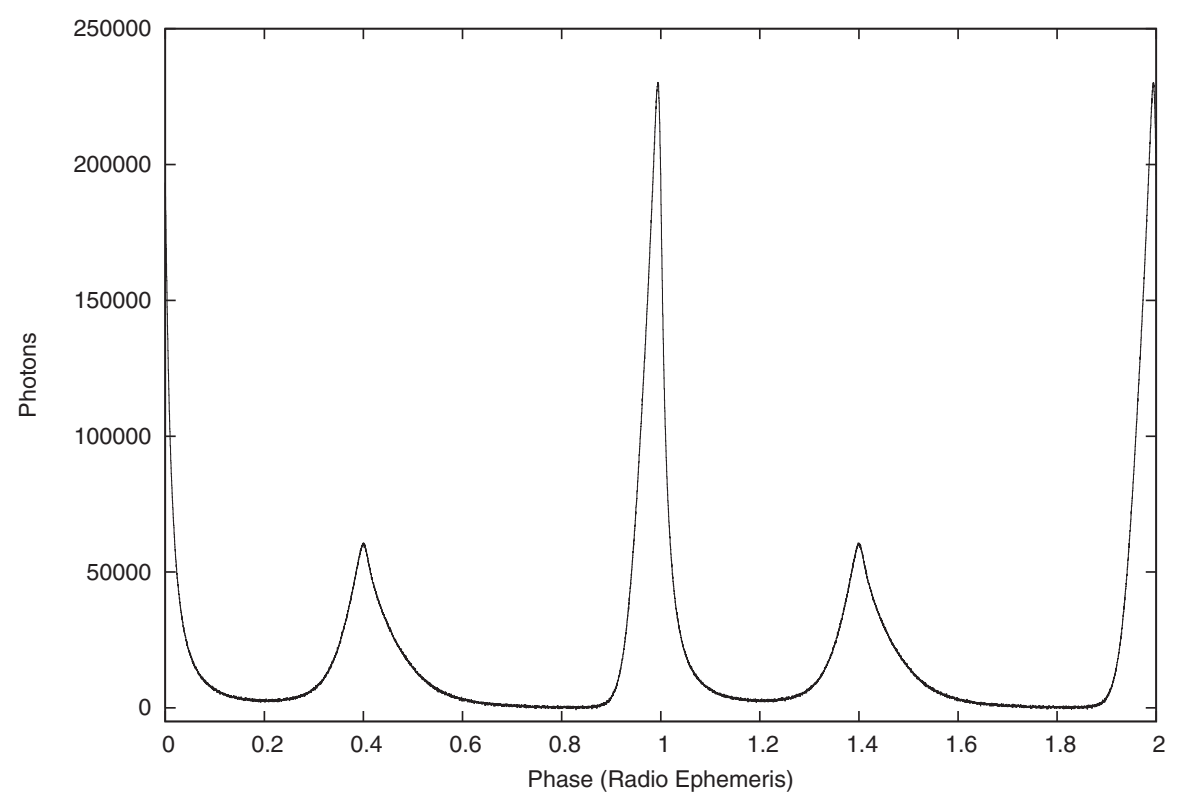

Figure 3. The Crab pulsar light curve, obtained by binning the IquEYE data using 5000 phase bins.

measure at the time. Fig. 3 shows the quality of the light curve obtained. In a few hours we reproduced the JB ephemerides to the picosecond level. Of all optical instruments, IquEYE on the NTT provided the best timing of photon arrival times.

\section{References}

Dravins, D., et al. 2005, QuantEYE. Quantum Optics Instrumentation for Astronomy, ESO document: OWL-CSR-ESO-00000-0162

Naletto, G., et al. 2009, A\&A, 508, 531-539 